\title{
Reversing defective lysosomal transport
}

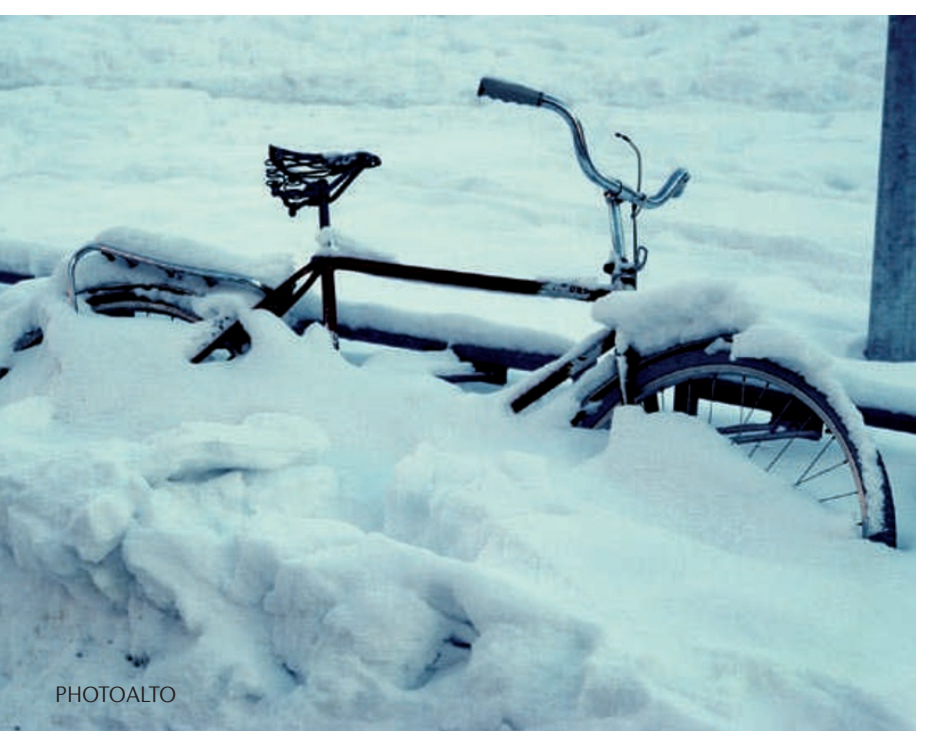

A recent study has shown that a commonly used cyclodextrin compound could have therapeutic effects in Niemann-Pick type C (NPC) disease. This condition - characterized by progressive liver disease and neurodegeneration - is largely caused by an inactivating mutation of NPC1, which normally aids the movement of unesterified cholesterol from the endosomal and lysosomal compartment to the cytosolic compartment of cells.

One potential way to overcome defective transport would be to deliver a compound into cells that reverses the transport defect in the late endosomal and lysosomal compartment, and allows the sequestered cholesterol to move into the metabolically active pool, where it could be metabolized and secreted through normal mechanisms. The authors used mice lacking the gene that encodes NPC1 $\left(N p c 1^{-/-}\right)$ as a model to investigate whether such a manipulation might be possible using the sterol-binding agent, 2-hydroxypropyl- $\beta$ cyclodextrin (2HP- $\beta-\mathrm{CyD})$.

After confirming earlier work in which $2 \mathrm{HP}-\beta-\mathrm{CyD}$ markedly prolonged the lifespan of $\mathrm{Npcl}^{-1-}$ mice, the authors investigated the acute effects of administration of the compound on sterol metabolism in 1 week-old mice. $2 \mathrm{HP}-\beta$-CyD had marked effects in $\mathrm{Npc1}^{-/}$animals: the increased rate of sterol synthesis was suppressed, the concentration of cholesterol decreased, the level of cholesteryl ester increased 14 -fold and total sterol concentration in the liver decreased. In addition, $2 \mathrm{HP}-\beta$ $\mathrm{CyD}$ lowered sterol concentrations in brain, liver and whole animal and returned the rates of synthesis to those seen in the untreated wild-type animals.

Next, it was shown that $2 \mathrm{HP}-\beta$ CyD treatment induced changes in the two regulatory systems in the cytosolic compartment that respond to variations in the size of the metabolically active sterol pool. The target genes of sterol regulatory element 2 and liver $\mathrm{X}$ receptor were modulated in the liver and brain of
$N p c 1^{-1-}$ mice following $2 \mathrm{HP}-\beta-\mathrm{CyD}$ administration. In addition, the compound reduced the overexpression of a number of inflammatory factors that lead to the presence of activated macrophages in NPC disease.

Studies were then carried out to identify the long-term effects in 49-day-old adult mice that had been given a single dose of $2 \mathrm{HP}-\beta-\mathrm{CyD}$ at 7 days of age. Six weeks after administration of $2 \mathrm{HP}-\beta-\mathrm{CyD}-$ when none of the compound was retained in the body - the concentration of cholesterol was still reduced in the liver, kidney and spleen, and the whole-animal cholesterol pool was reduced even though synthesis was increased. Moreover, liver function tests were markedly improved and the level of neurodegeneration in the brain was significantly reduced. Finally, at 49 days of age, the external sterol balance in the $2 \mathrm{HP}-\beta-\mathrm{CyD}$ treated $\mathrm{Npc1}^{-1-}$ mice had returned to levels seen in the untreated animals.

Although miglustat, an inhibitor of glucosylceramide synthase and a-glucosidase, has recently been approved within the European Union for the treatment of progressive neurological manifestations in NPC disease, treatment options for the condition are limited. Even though further studies are needed to elucidate the best dosing schedule and route of $2 \mathrm{HP}-\beta-\mathrm{CyD}$ administration, this paper demonstrates that a compound routinely used in drug formulations - and therefore safe in humans - could have therapeutic potential in NPC disease.

Charlotte Harrison

ORIGINAL RESEARCH PAPER Liu, B. et al. Reversal of defective lysosomal transport in NPC disease ameliorates liver dysfunction and neurodegeneration in the $n p c 1^{--}$mouse. Proc. Natl Acad. Sci. USA 26 Jan 2009 (doi: 10.1073/pnas.0810895106) 